\title{
Specific Removal of C-Reactive Protein by Apheresis in a Porcine Cardiac Infarction Model
}

\author{
Anna Christine Slagman ${ }^{\mathrm{a}}$ Christopher Bock ${ }^{\mathrm{b}}$ Hassan Abdel-Aty ${ }^{\mathrm{e}}$ Birgit Vogt $^{\mathrm{b}}$ \\ Frank Gebauer $^{\mathrm{b}}$ Gunnar Janelt ${ }^{\mathrm{b}}$ Franziska Wohlgemuth ${ }^{\mathrm{b}}$ Rene Morgenstern ${ }^{\mathrm{b}}$ \\ Gülcan Yapici $^{b}$ Astrid Puppe ${ }^{b}$ Diethelm Modersohn ${ }^{a}$ Dörte Mans ${ }^{a}$ Timo Jerichow ${ }^{a}$ \\ Sascha Ott ${ }^{d}$ Rudolf Kunze ${ }^{f}$ Wieland Schrödl ${ }^{g}$ Christina Janko ${ }^{\text {h }}$ Martin Hermann ${ }^{\text {h }}$ \\ Joachim R. Kalden ${ }^{\mathrm{h}}$ Peter Kern ${ }^{\mathrm{j}}$ Hans Parsch ${ }^{\mathrm{i}}$ Michael Kirschfink ${ }^{\mathrm{k}}$ Jeanette Schulz-Menger ${ }^{\mathrm{e}}$ \\ Rainer Röttgen ${ }^{c}$ Juliane K. Unger ${ }^{d} \quad$ Ulrich Frei $^{b}$ Ralf Schindler ${ }^{b}$ Martin Möckel ${ }^{a} \quad$ Ahmed Sheriff $^{b}$ \\ ${ }^{a}$ Emergency Medicine Unit, Department of Cardiology, b Department of Nephrology and Internal Intensive Care \\ Medicine, 'Department of Radiology, ${ }^{\mathrm{d}}$ Department for Animal Care, Charité Berlin, ${ }^{\mathrm{e} C a r d i a c}$ MRI Team, \\ Franz-Volhard-Klinik, Charité Berlin, Helios Clinic Berlin-Buch, and ${ }^{\mathrm{f}}$ Office Campus Max Delbrück Centrum, Berlin, \\ IInstitute of Bacteriology and Mycology, University of Leipzig, Leipzig, h Department of Internal Medicine III, \\ Institute for Clinical Immunology and Rheumatology, and 'Central Laboratory, University Hospital Erlangen, \\ Erlangen, ${ }^{j}$ Capio Franz von Prümmer Klinik, Bad Brückenau, and k Department of Immunology, University of \\ Heidelberg, Heidelberg, Germany
}

\section{Key Words}

Acute myocardial infarction - Acute phase protein •

Adsorber $\cdot$ Apheresis $\cdot$ C-reactive protein

\begin{abstract}
Background: C-reactive protein (CRP) is a possible causative factor of the destructive processes observed during the weeks after myocardial infarction. Methods: We developed a clinically relevant animal model including the removal of CRP from blood plasma utilizing a specific CRP adsorber and the visualization of the infarct scar in the living animal by cardiovascular magnetic resonance imaging as a tool to investigate the impact of CRP after acute myocardial infarction. Results: We describe the facets of this model system and kinetics of clinical blood parameters like CRP and troponin. In addition, we demonstrate the potency of CRP apheresis reducing CRP levels by $\sim 70 \%$ in the established treatment system. Conclusion: We showed for the first time that
\end{abstract}

it is possible to conduct apheresis at the following 2 days after acute myocardial infarction in a porcine infarction model and to analyze the infarct by cardiovascular magnetic resonance imaging at day 1 and 14 . Copyright $\odot 2010$ S. Karger AG, Basel

C-reactive protein (CRP), a classical acute phase protein, has been extensively studied as a systemic marker of inflammatory processes. During acute myocardial infarction (AMI), the circulating CRP concentration increases and its level predicts the outcome of AMI [1-3]. However, there is still a controversy whether CRP is only a marker or a risk factor, contributing causative to myocardial damage after AMI.

In rats, administration of human CRP after ligation of the coronary artery reproducibly enhanced infarct size by up to $40 \%[4,5]$. They received $40 \mathrm{mg} / \mathrm{kg}$ of isolated pure human CRP by intraperitoneal injection $1 \mathrm{~h}$ after coro-

\section{KARGER}

Fax +41613061234 E-Mail karger@karger.ch www.karger.com
(C) 2010 S. Karger AG, Base

$0253-5068 / 10 / 0313-0009 \$ 38.00 / 0$

Accessible online at:

www.karger.com/bpu
Dr. Ahmed Sheriff

Charité-Universitätsmedizin Berlin

Hessische Strasse 3-4

DE-10115 Berlin (Germany)

Tel. +49 30450513 326, Fax +49 30450513 936, E-Mail ahmed.sheriff@charite.de 
Fig. 1. Time schedule of the treatment. The interventions during the first $50 \mathrm{~h}$ of the experiment are highlighted. The arrows indicate times of blood collection.

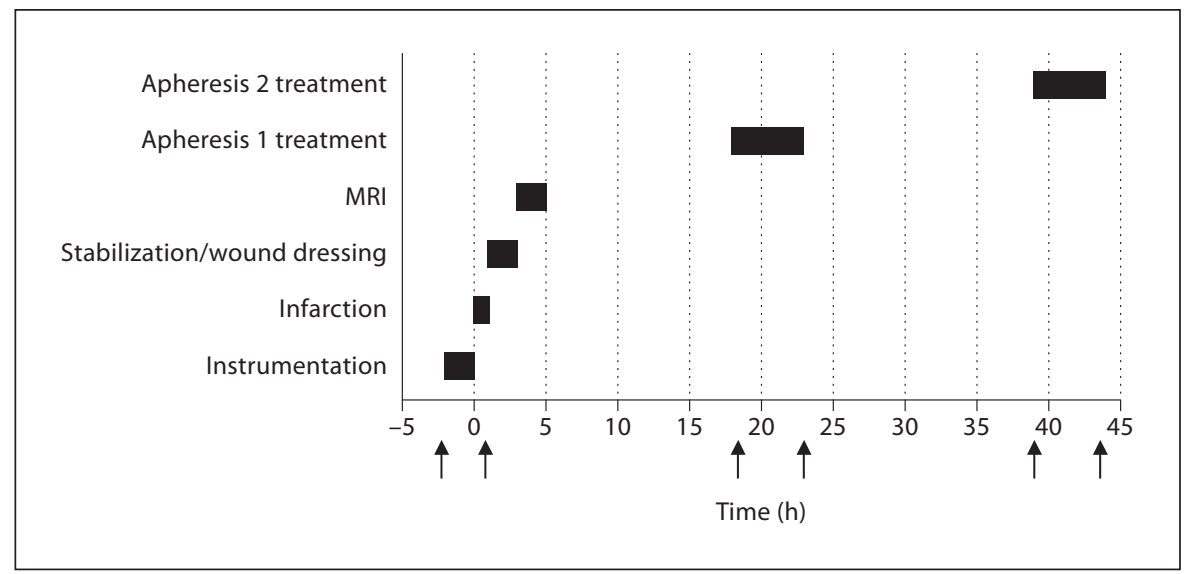

nary artery ligation and received further daily injections of the same dose of human CRP during the next 5 days. In vivo concentrations of close to $600 \mathrm{mg} / \mathrm{l}$ of human CRP were achieved. Complement depletion abrogated the infarct-enhancing effect. Therefore, human CRP and complement activation are major mediators of ischemic myocardial injury, and they are potential therapeutic targets in coronary heart disease.

There is, however, no suitable animal model for removal of endogenous CRP induced by myocardial infarction. One obstacle is the different regulation and function of CRP between animals and humans. In the common mouse and rat models, CRP is regulated differently and has other functions than in humans $[6,7]$. Fortunately, in pigs regulation and function of CRP is similar to humans [8]. In addition, pigs are known for the similarity of their cardiac system to the human cardiac system. Furthermore, pigs provide sufficient body mass for an extracorporeal blood circuit established for adult patients allowing the use of clinical size adsorbers $[9,10]$. Removal of CRP can be achieved by adsorption to phoshorylcholinecoupled Sepharose as performed in laboratory purification procedures.

Cardiovascular magnetic resonance (CMR) imaging has emerged as a powerful tool to quantify the myocardial damage following AMI. The so-called late gadolinium enhancement allows an accurate quantification of myocardial necrosis/scar matching that of histopathology [11], and justifying its use to monitor the infarct sizereducing effect of novel reperfusion strategies. In contrast to histopathological studies, the method offers in vivo assessment allowing monitoring these effects in the living animals over time and relating it to left ventricular remodeling and function.
The aim of the current study was to establish a model of AMI in pigs in combination with an extracorporeal depletion of CRP.

\section{Methods}

\section{Study Design}

The study was designed to establish specific CRP apheresis after myocardial infarction. The care and treatment of the animals (female pigs, German landrace, body weight $35-50 \mathrm{~kg}$ ) were in accordance with German requirements. The study protocol (Reg. No.: 0283/07) was approved by the University Animal Care Committee and the federal authorities for animal research in Berlin, Germany. The experiments were performed at the facilities of the Department of Experimental Medicine (certified by ISO9001). The principles of laboratory animal care were followed with respect to the guidelines of the European and German societies of laboratory animal sciences.

After myocardial infarction, infarct size was assessed applying CMR imaging (CMRI). 18-21 h after infarction, the first apheresis was performed, followed by the second apheresis 22-24 h later (fig. 1). Blood samples for CRP measurement were drawn before and after infarction, before and after apheresis, and on days 4-7.

Four pigs were euthanized after CMRI by heart arrest (potassium chloride injection), and the hearts were removed for histological examination (infarct size and area at risk, AAR).

\section{Anesthesia, Instrumentation and Myocardial Infarction}

Prior to the experiments, a prophylactic antibiotic treatment was indispensable to minimize the risk of latent or subclinical respiratory and musculoskeletal infections, which might become apparent during postinfarction stress.

On the day of the experiment, i.m. premedication was performed by injection of azaperon, atropine sulphate, ketamine and xylazine hydrochloride. After preoxygenation, induction of general anesthesia was performed by i.v. injection of propofol followed by orotracheal intubation. Anesthesia was maintained with inhaled isoflurane $1 \%$, fentanyl $60 \mu \mathrm{g} / \mathrm{kg} / \mathrm{h}$ and mechanical 
ventilation with oxygen-enriched air $\left(\mathrm{FiO}_{2}>0.8\right)$. Oxygenation $\left(\mathrm{SpO}_{2}\right)$, body temperature, electrocardiogram and blood pressure were continuously measured as well as intermittent central venous pressure. Blood gas analysis and monitoring of glucose, lactate, base excess, $\mathrm{Ca}^{2+}, \mathrm{Mg}^{2+}, \mathrm{K}^{+}$and $\mathrm{Na}^{+}$were conducted frequently.

After arterial and venous access, an uncoated dual lumen silicon catheter for apheresis was placed into the vein with the tip close to the right atrium during anticoagulation with heparin (5,000 I.E.).

Closed-chest anteroseptal myocardial infarction was created by transient occlusion of the D2 branch of the RIVA (ramus interventriculars anterior). After systemic administration of $5 \mathrm{mg}$ bolus of acetylsalicylic acid, a coronary balloon catheter was positioned over a guide wire and inflated up to 6 bar. Correct localization of the inflated balloon and proper occlusion was visualized by angiography. To confirm the patency of the artery, a second angiography was performed after deflation of the balloon.

During reperfusion, the circulation of the animal was stabilized, using $300 \mathrm{mg}$ amiodarone or $1 \mathrm{mg}$ norepinephrine if needed to avoid ventricular arrhythmia. The anesthesia was switched to analgosedation with ketamine and propofol during CMRI. Postoperative analgesia was ensured by fentanyl plaster and if necessary $300 \mu \mathrm{g}$ buprenorphine i.m.

All animals received $100 \mathrm{mg}$ acetylsalicylic acid daily until the end of the experiment.

\section{Apheresis}

Adsorber for CRP Depletion

The CRP adsorber comprises $55 \mathrm{ml}$ of Sepharose Fast Flow 4 (GE Health Care - BioScience, Uppsala, Sweden) covalently coupled with approximately $30 \mu \mathrm{mol}$ phosphorylcholine per gram soaked matrix via a C6 spacer. The obtained matrix was autoclaved and filled into previously fitted and ethylene oxide sterilized cartridges, equal to the one used in the immunoadsorber Immunosorba $^{\circledR}$ (Fresenius Medical Care, Bad Homburg, Germany). Filling was performed using a closed system composed of sterile medical device components in a previously decontaminated laminar flow cabinet. Filled adsorbers were finally flushed with phosphate-buffered saline (PBS) pH 7.3 containing $0.01 \% \mathrm{NaN}_{3}$ (Serag Wiesner $\mathrm{KG}$, Naila, Germany), capped and stored at $2-8^{\circ} \mathrm{C}$ until use.

\section{Apheretic Treatment}

Apheresis was performed at days 2 and 3 after infarction under general anesthesia with ketamine and propofol. During apheresis, the twofold plasma volume estimated by body weight and hematocrit status was processed.

CRP depletion was performed with a regenerative single-adsorber-system, after separation of plasma and consecutive perfusion of the plasma through the CRP adsorption unit. Immediately after processing, the treated plasma was returned into the pig via the venous air trap of the plasma separation circuit. In contrast to commonly used double-adsorber systems, plasma was bypassed during the regeneration phase. For regeneration, plasma was displaced by $0.9 \%$ sodium chloride solution (DeltaSelect, Pfullingen, Germany) followed by $\mathrm{pH}$ shift with glycine/ $\mathrm{HCl} \mathrm{pH}$ 2.8 (Serag Wiesner KG). For neutralization, PBS pH 7.3 (Serag Wiesner KG) was used followed by $0.9 \%$ sodium chloride before the next loading with plasma. All solutions and disposables used were sterile, and endotoxin-free medical devices intended for extracorporeal treatments. Plasma separation and flow monitoring were performed by the BM11 (Edwards Lifescience, Irvine, Calif., USA) using the plasma filter BT 900 B (Dideco, Mirandola, Italy). Flow of plasma and solutions during treatment was controlled by an ADAsorb apheresis control unit (medicap clinic GmbH, Ulrichstein, Germany) with a specially adapted software module for automatic plasma flow management. Per treatment, 6-8 treatment cycles were performed. Mean plasma flow through the adsorber was $25-30 \mathrm{ml} / \mathrm{min}$, while the blood flow to the primary system ranged from 150 to $180 \mathrm{ml} / \mathrm{min}$. After treatment, adsorbers were preserved by rinsing with PBS containing $0.01 \%$ sodium azide (Serag Wiesner KG) after the last regeneration phase.

\section{Anticoagulation during Apheresis}

During the preparation of the apheretic system, the plasma filter was primed with 5,000 IU heparin not injected into the animal. The animal was anticoagulated with a systemic bolus of 1,0002,000 IU followed by constant perfusion of 500-3,000 IU per hour. Maintenance of anticoagulation was monitored by frequent activated coagulation time (ACT) measurement with ACT $>180 \mathrm{~s}$.

\section{Cardiac Magnetic Resonance}

CMR Image Acquisition

CMR studies were accomplished in a 1.5-tesla system (Signa CV/i; GE Medical Systems, Milwaukee, Wisc., USA). Localization was performed using non breath hold real time images of true anatomical axes of the heart. Breath hold steady-state free precession images (TR $3.7 \mathrm{~ms}$, TE $1.7 \mathrm{~ms}, 30$ phases) were then acquired in short and long axis (2-, 3- and 4-chamber views) orientations. The short axis slices covered the entire left ventricular myocardium from the mitral valve to the apex (slice thickness: $8 \mathrm{~mm}$ with no gap). A contrast-enhanced inversion recovery gradient echo sequence was then performed in the same slice locations using the same thickness/gap parameters as steady-state free precession images (TR $7.1 \mathrm{~ms}$, TE $3.1 \mathrm{~ms}$, matrix $256 \times 192$, TI: optimized to null remote myocardium signal intensity) 10 min after i.v. injection of Gd-DTPA.

\section{CMR Image Analysis}

All analyses were performed using dedicated software (CMR $42^{\circledR}$, CIRCLE International Ltd., Calgary, Alta., Canada). For late enhancement, after tracing the endocardial and epicardial contours, regions of interest were manually drawn within the normal myocardium, and the software automatically delineated areas of high signal intensity based on different user-identified thresholds of 5 standard deviations above that of the average normal myocardial signal intensity (remote myocardium was defined as that with preserved systolic function and absence of late gadolinium enhancement). The volume of myocardium exhibiting high signal intensity was then quantified and related to that of the slice volume. These volumes were multiplied by the specific gravity of the myocardium (1.05) to calculate the mass in grams. Areas of microvascular obstruction (no reflow) were considered as part of the infarct zone.

Left Ventricle Function and Mass

The endocardial and epicardial contours were manually traced including the papillary muscles and trabeculations both in systole and diastole. Left ventricle (LV) end-diastolic and endsystolic volumes were quantified as well as the ejection fraction and the LV volume and mass. 
CRP ELISA Systems

Porcine CRP (pCRP) was determined by three different ELISA test systems, subsequently termed Dako, R\&D and Tridelta. The Dako-ELISA used a procedure as described elsewhere in detail [12]. The primary antibody (polyclonal rabbit anti-human CRP; $1 \mathrm{mg} / \mathrm{ml}$; Dako, Glostrup, Denmark) was diluted 1:5,000 in carbonate-bicarbonate buffer $(0.1 \mathrm{M}, \mathrm{pH} 9.6)$. For detection, one batch of the antibody $(7 \mathrm{mg} / \mathrm{ml})$ was labeled with peroxidase and subsequently diluted 1:5,000. Detection was performed using tetramethylbenzidine dihydrochloride (Sigma Aldrich, Munich, Germany). Highly purified pCRP served as a control. Detection limit of this assay was $15 \mathrm{ng} / \mathrm{ml}$.

A second ELISA used two monoclonal antibodies obtained from $R \& D$ (R\&D Systems, Wiesbaden, Germany). The primary antibody was diluted 1:100 (monoclonal anti-human/mouse/porcine CRP; R\&D Systems), the second biotin-labeled antibody was diluted 1:200 (biotinylated anti-human CRP; R\&D Systems). For detection, peroxidase-coupled streptavidin (dilution 1:200) was used (R\&D Systems). This assay used a human CRP standard (Sigma Aldrich). Detection limit of this assay was $40 \mathrm{ng} / \mathrm{ml}$.

The Tridelta ELISA was performed according to the manufacturer's instructions (CRP porcine; Tridelta Development Ltd., Maynooth, Ireland). Detection limit of this assay was $23 \mathrm{ng} / \mathrm{ml}$.

\section{Blood Analysis}

Troponin T and I, aspartate aminotransferase (AST) and serum albumin were measured in the Institute for Laboratory Medicine and Pathobiochemistry, Charité, Berlin, Germany, using standard procedures. Protein was determined using RotiNanoquant (Carl Roth GmbH, Karlsruhe, Germany) according to the manufacturer's instructions. C3b determination was performed by a cross-reactive assay for human C3b (Binding Site, Schwetzingen, Germany) according to the manufacturer's instructions.

\section{SDS Gel Electrophoresis and Western Blot}

SDS gel electrophoresis and Western blot analysis were performed using kits and devices from Invitrogen (Invitrogen, Carlsbad, Calif., USA) according to the manufacturer's instructions. SDS gel electrophoresis was performed using the XCell SureLock $^{\text {TM }}$ System with NuPage ${ }^{\circledR} 12 \%$ Bis-Tris-Gel, SeeBlue ${ }^{\circledR}$ PreStained Standard and NuPage MES SDS running buffer. Gels were stained using SimplyBlue ${ }^{\mathrm{TM}}$ SafeStain. For Western blot analysis, gels were run with MagicMark ${ }^{\mathrm{TM}} \mathrm{XP}$ standard and blotted on Invitrilon ${ }^{\mathrm{TM}}$ PDVF membranes using the blotting device of the XCell SureLock ${ }^{\mathrm{TM}}$ system. For detection, the Western Breeze ${ }^{\circledR}$ Kit for rabbit antibodies was used. The specific polyclonal antibody mixture for detection of CRP was rabbit anti-human CRP (Dako, Glostrup, Denmark) diluted 1:10,000. For serum amyloid $\mathrm{P}$ (SAP) detection, a rabbit anti-human/-mouse/-rat SAP antibody (Novus Biologicals Inc., Littleton, Colo., USA) in a 1:4,000 dilution was used.

\section{Histological Examination}

For histological examination of the removed heart, the D2 branch was ligated at the site of the previous occlusion. Through a coronary catheter, 1\% Evans Blue (Applichem GmbH, Darmstadt, Germany) was injected to inversely mark the AAR. Thereafter, the heart was cut into transversal slices of $8 \mathrm{~mm}$ thickness. These slices were incubated in $2 \%$ triphenyltetrazolium chloride
(Sigma Aldrich) for $10 \mathrm{~min}$ to discriminate between vital and necrotic cells within the AAR. Based on digital pictures, the AAR, necrosis, and the surface of the LV were measured by computerized planimetry (ImageJ 1.410, NIH). The percentage of the ARR and necrosis in relation to the LV was calculated for each slice.

\section{Results}

\section{Antibiotics}

Our first experiments showed a broad range of CRP levels in pigs after the routine antibiotic treatment for 2 days ( $\mathrm{n}=16$; mean $22.27 \mu \mathrm{g} / \mathrm{ml}$ ); therefore, we extended antibiotic treatment for one week prior to experiments to reach low levels of CRP $(n=10$; mean $=5.86 \mu \mathrm{g} / \mathrm{ml})$.

\section{Anesthesia}

During establishment of the porcine animal model, we encountered a number of particular challenges in the anesthesiological and postoperative management of the animals. Experimental interventions had a tight time schedule (see also fig. 1), which led to a decreasing physiological status of the pigs. Especially problematic were the repeated anesthesia for often more than $5 \mathrm{~h}$ (8-9 $\mathrm{h}$ at day one), the daily loss of blood and plasma as well as the reduced intake of water and food. Repeated orotracheal intubation and mechanical ventilation increases the risk of pneumonia and atelectasis (primarily in pigs that often initially were not completely healthy).

Frequent analysis of glucose and ions was performed. The physiological values of $\mathrm{Ca}^{2+}, \mathrm{Mg}^{2+}, \mathrm{K}^{+}$and $\mathrm{Na}^{+}$in the pig differ slightly from humans; therefore, adjustment of infusions were necessary. Infusions were balanced under consideration of central venous pressure and diuresis to avoid increasing cardiac preload. In the case of early development of pulmonary edema, furosemide was needed. During apheresis, some animals demonstrated sufficient breathing patterns and a good oxygenation but hyperkapnia occurred. In these cases, THAM buffer and, if necessary, mechanical ventilation was used to prevent respiratory acidosis.

\section{Variability of Infarction Size}

Our next issue was to establish a reproducible infarction procedure to minimize the variability. We aimed for an infarction size of $10 \%$ of the LV. This was achieved by occluding the D2 branch of the RIVA for $60 \mathrm{~min}$. The placement of the balloon in this branch was adjusted according to the specific anatomy of the heart of the individual pig. This resulted in the anticipated infarction size 
of $10 \pm 2.11 \%(n=14$; fig. $2 \mathrm{a})$. Infarction size was assessed by MRI analysis and confirmed by histological staining of the area of infarct ( $n=4$; fig. $2 b)$.

In six pigs, blood was drawn before and up to $3 \mathrm{~h}$ after the infarct. As expected, $1.5-3 \mathrm{~h}$ after infarction, troponin T, troponin I and AST (not shown) were increased in the infarcted animals $(\mathrm{n}=4)$. A noninfarcted control showed no increase $(n=2)$. CRP started to rise 5-6 h after start of surgery ( $4-5 \mathrm{~h}$ after AMI), independent of infarction (not shown).

\section{CRP Quantification}

CRP quantification was performed using ELISA [12] (Dako) as established by W. Schrödl. First experiments showed a 5- to 10 -fold difference to the commercially available assay kit from Tridelta (fig. 3a) as also reported by others [13]. The quantification was therefore controlled using a second ELISA (fig. 3a) also based on crossreacting antibodies to human CRP (R\&D Systems). This ELISA gave similar results to the assay according to $\mathrm{W}$. Schrödl [12]. Despite the differences to the Tridelta ELISA in the absolute amount of CRP, the relative kinetics in the three assay systems were the same (fig. 3b). We used isolated pig CRP as a standard for the Dako ELISA and human CRP standard serum for the R\&D-based ELISA. The Tridelta standard solution contained no CRP protein, as analyzed by SDS gel (not shown).

\section{CRP Removal}

Blood was drawn before and after infarction, before and after apheresis, and each day up to day 7 and on day 14. On day 4-7, blood was drawn through the Hickman catheter from the unsedated animals. Before the experiment, the animals were trained to allow the handling of the catheter by the veterinarians. As shown in figure 4, a good reduction in CRP levels was achieved using specific CRP apheresis. A slight rebound can be seen on day 3, but CRP does not reach the CRP level of the sham control. A slight decrease in CRP levels was also observed in shamtreated animals. This is due to the loss of blood and to substitution by infusions. Overall protein and albumin content of the blood reflected this reduction (data not shown).

A fraction of glycine/ $\mathrm{HCl}$ used for regeneration of the adsorber was collected after passing the adsorber. This corresponds to the elution fraction in a laboratory setting of affinity chromatography. The elution was analyzed by CRP ELISA, Bradford assay and SDS gel electrophoresis (fig. 5). CRP was enhanced in the elution fraction in comparison to serum. CRP content was $5-15 \%$ of
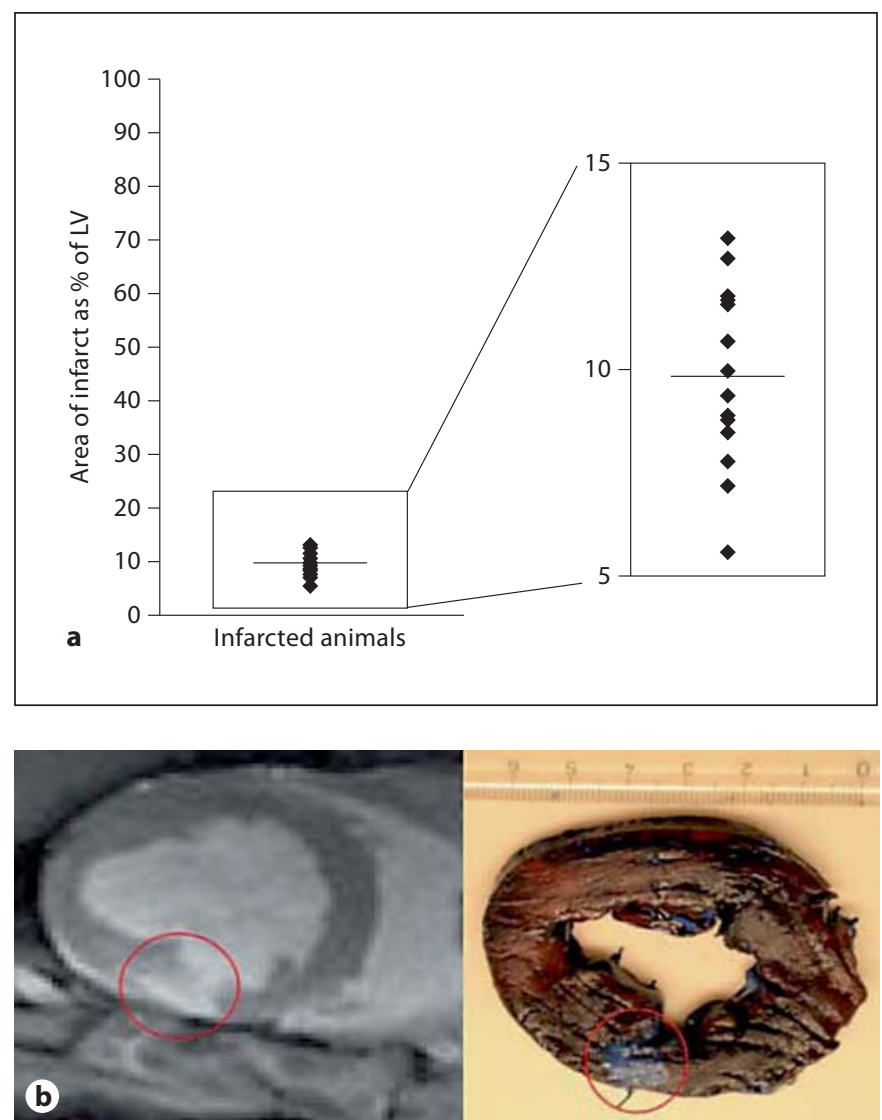

Fig. 2. Infarct sizes. a The distribution of infarct size in the LV was determined using CMRI $(n=14)$. $\mathbf{b}$ Histological analysis of the infarct in the LV on day 14. Shape and size of the areas of infarct are similar in both imaging methods. One representative example is shown $(\mathrm{n}=5)$.

total protein in the elution fraction, while its amount was $0.17 \%$ of total protein in serum. Each elution fraction showed a strong CRP band in the SDS gel. The identity of this protein as CRP was confirmed by Western blot analysis. In contrast, SAP, another pentraxin, could not be detected.

Additionally, blood plasma of the pigs was analyzed by SDS gel electrophoresis and Coomassie brilliant blue staining before and after apheresis. We analyzed different protein amounts to get univocal information. CRP could not be detected in the plasma before or after apheresis, which indicates that the CRP concentration was below the detection limit of this method and that we enriched CRP in the apheresis fractions (fig. 5a). The pattern of the main protein bands before and after apheresis was identical, showing that the apheresis did not crucially decrease other main proteins (fig. 5a). 
Fig. 3. Comparison of three different ELISAs for quantification of pCRP. a Mean and SD of CRP level of an infarcted pig (VV1). The Dako assay and the assay using the $R \& D$ antibodies result in nearly the same absolute CRP levels. The levels according to the Tridelta assays are 5 times higher ( $\mathrm{n}=2$ for R\&D and Tridelta; $\mathrm{n}=4$ for Dako). b The relative CRP kinetics were very similar in all assays. CRP levels of an infarcted pig (VV5) undergoing CRP apheresis were calculated as relative units. The level before infarction was set as 1 and the following values were related to it.

Fig. 4. CRP is reduced after specific CRP apheresis. Shown is the development of CRP values in $\mathrm{mg} / \mathrm{l}$ of 2 animals. One animal was subjected to CRP apheresis (verum; VV9), the other to sham apheresis without adsorber (sham; VV8). Specific apheresis reduces the level of CRP permanently. In the sham-treated animal, CRP loss in the first $48 \mathrm{~h}$ is due to hemodilution (blood and serum loss; volume management).
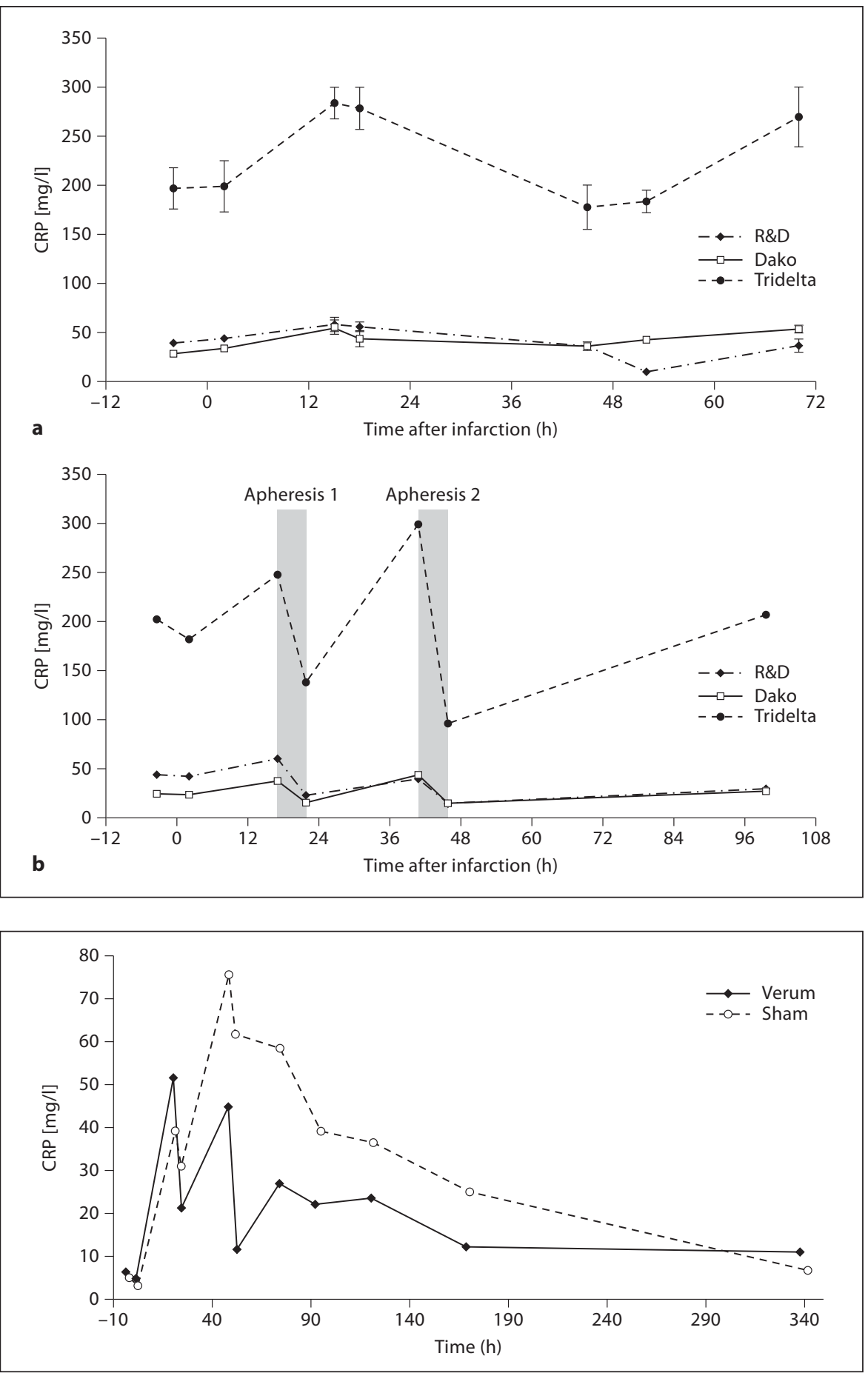

No complement (C3 and C5b-C9) activation due to apheresis was observed (not shown) which exceeds activation caused by protein A-Sepharose as used in the immunoadsorber Immunosorba (Fresenius Medical Care) observed during immunoglobulin apheresis in pigs (pre- liminary experiment, not shown). The analyses of eluted fractions by SDS gel electrophoresis and ELISA after apheresis showed an enrichment of CRP $(5-15 \%$ in the elution fractions vs. $0.17 \%$ in serum; enrichment factor: 29-88). 
Fig. 5. Analysis of the apheresis elutions by SDS gel electrophoresis and Western blot analysis. a SDS gel electrophoresis of the elution fractions from two aphereses of one animal and the corresponding plasma before and after the first apheresis. The plasma lanes contain $5 \mu \mathrm{g}$ protein each, the elution lanes 3.5-5 $\mu$ g. No CRP can be detected in the plasma, whereas the elutions show a distinct CRP band. Shown is one representative animal $(n=6)$. Purified pCRP was used as a standard and is slightly visible; the CRP band is indicated by the arrow. Molecular weight marker: SeeBlue Pre-Stained Standard (Invitrogen). b Western blot analysis of the same elution fractions and plasma. Shown is the blot using the anti-hCRP antibody and purified pCRP as positive control. The CRP band is prominent in each lane of the elutions and can also be detected in the plasma samples. The plasma before apheresis shows a stronger band than after apheresis.
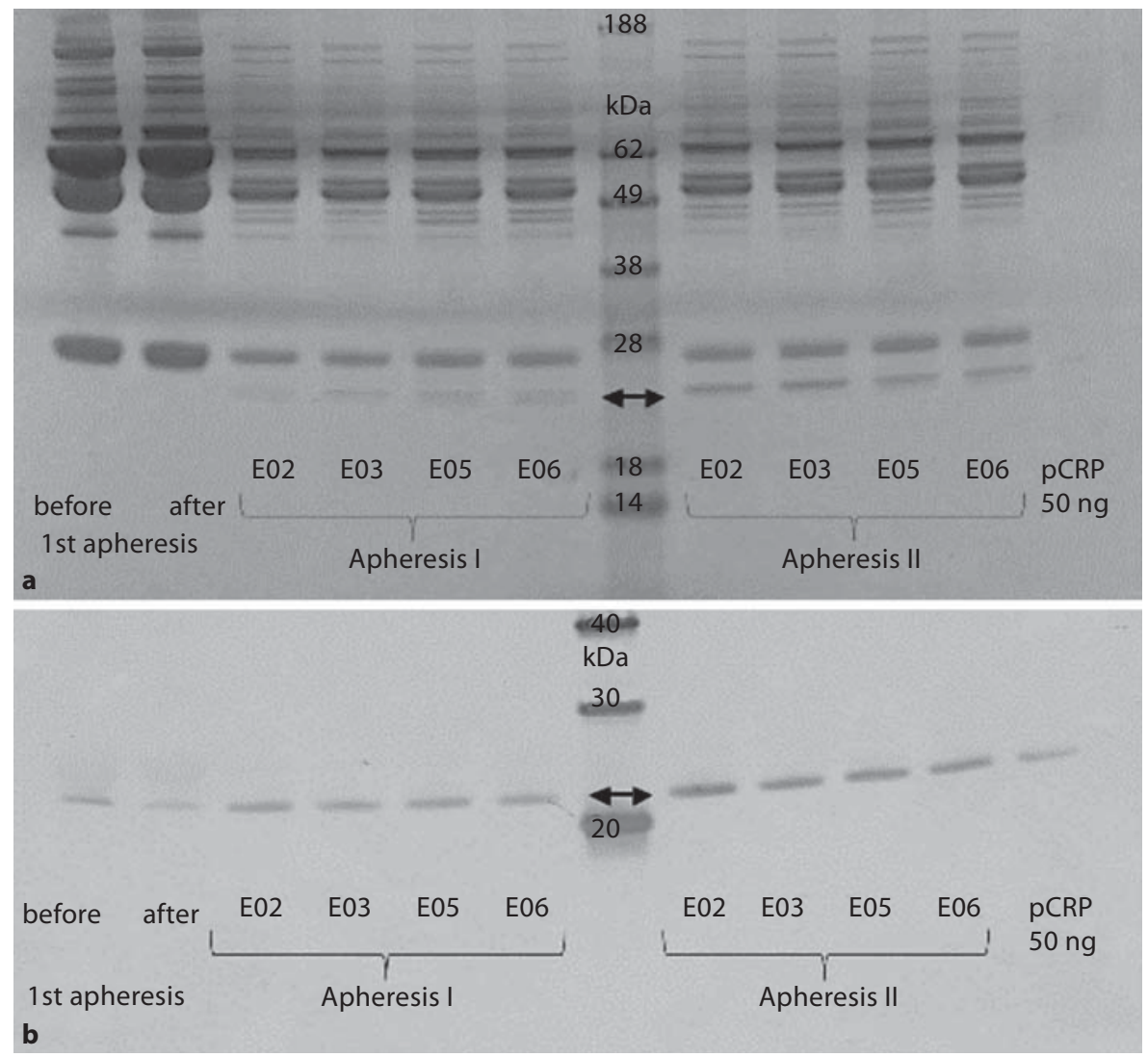

A Western blot analysis of elution fractions and plasma showed that the CRP band is prominent in each lane of the elutions and can also be detected in the plasma samples. As expected, the plasma before apheresis showed a stronger band than the plasma after apheresis (fig. 5b). Additionally, we analyzed the concentration of a different pentraxin, namely SAP, to demonstrate that the adsorber is specific for CRP by using Western blot analysis. We were not able to detect SAP in the elution fractions (not shown).

Overall protein and serum albumin levels were similar in animals with verum or sham apheresis as well as in untreated controls (not shown). Therefore, we conclude that we were able to establish a feasible and reproducible model of infarction followed by specific apheresis.

\section{Discussion}

Despite AMI being one of the major causes of death, regarding the pathophysiology of remodeling during days and weeks after AMI, many open questions remain. One of these questions is the contribution of acute-phase proteins, especially CRP, to destruction processes in the area of infarction.

A major determinant for IL-6 production is the level of adiposity, especially central abdominal obesity [14], reflecting production of proinflammatory cytokines like IL- 6 by macrophages associated with such adipose tissue and by the adipocytes themselves. CRP and IL-6, a potent inducer of CRP, are correlated to subacute and chronic infarct size [15].

CRP has characteristics that may contribute to the pathophysiology of AMI, and it has been shown in animal models that high concentrations of CRP increase the area of infarct $[4,5]$. CRP has been used as a prognostic marker for decades [1-3] but its active contribution has been unclear.

Pepys et al. [5] achieved the reduction of this enhanced damage by specific molecular blocking of human CRP using 1,6-bis(phosphocholine)-hexane. This small molecule has a short half-life (90 min) and had to be administered before infarction and constantly afterwards. The rats also received injections of human CRP daily, which, in contrast to rat CRP, activates the rat complement sys- 
tems after binding dying or dead cells. This model shows the participation of human CRP in myocardial damage after AMI, but it allows no conclusion regarding the regulation of endogenous CRP in this setting. As Kitsis and Jialal [16] mentioned in an editorial to this article, the model lacks strong comparison to the clinical situation regarding the timing of drug administration, reperfusion, etc. As of today, no therapeutic option to specifically lower the CRP amount in humans in an acute care situation as encountered in AMI is available. Only unspecific apheresis like lipid apheresis, cascade filtration [17, 18] and fibrinogen apheresis [19] has been shown to remove CRP and other serum proteins in humans in addition to their intended use.

A renowned model for AMI is the closed chest infarction model in the pig. Here, we combined it with a specific apheresis of endogenous CRP. By placing the balloon in the D2 branch of the RIVA and an occlusion period of $60 \mathrm{~min}$, we achieved a highly reproducible infarct size of around $10 \%$ of the $\mathrm{LV}$ with a variation of $2 \%$.

As reported in humans, $1.5-3 \mathrm{~h}$ after infarction the troponins as well as AST started to rise in the infarcted pigs. Due to surgery, an increased CRP level was detected $6 \mathrm{~h}$ after start of the procedure, which equals $4-5 \mathrm{~h}$ after the start of the experimental infarction. The main peak of CRP occurred between 24 and $50 \mathrm{~h}$. This also resembled the situation in human patients where CRP rises between 4 and $6 \mathrm{~h}$ after infarction and peaks between 24 and $50 \mathrm{~h}[2,4,5]$. Therefore, the closed chest infarction model of the pig is a highly suitable model for CRP elimination after AMI.

Use of apheresis as a treatment form for other indications and removal of plasma proteins like immunoglobulins is safe and well documented in humans and may also be applied after infarction to limit the blood concentration of CRP. We conducted the first apheresis 16-21 h after infarction and, therefore, before the CRP level peaks. For further CRP reduction, a second apheresis was performed approximately $24 \mathrm{~h}$ later. We observed a slight rebound and/or redistribution of CRP on day 3. It will be interesting to figure out the relevance of such a rebound phenomenon with respect to the clinical outcome after AMI. In contrast to human patients, pigs need to be anaesthetized during apheresis. Repeated anesthesia presented the most relevant limitation of the model. Anesthesia had to be performed very skillfully, and the animals had to be monitored closely to avoid instability and sudden death. The described anesthesiological regime allowed the performance of major manipulations of the animals every day during a 3-day course. Animals lived thereafter until euthanization another 12-35 days under standard conditions without further complications.

Due to the required recovery of the animals after anesthesia, we could apply the first apheresis only as early as $16 \mathrm{~h}$ after infarction - which represents a further drawback of this model. Therefore, we cannot determine the outcome of earlier interventions.

We were able to draw blood from the unsedated animals using the Hickman catheter thanks to a consequent behavioral training and conditioning of the pigs before the experiment and due to their interest in tidbits. Further sedation would not have been possible. The overall procedure allowed the follow-up of the kinetics of blood parameters for 7 days.

CRP apheresis was well tolerated and did not show adsorber-related adverse effects. In particular, no relevant complement activation was observed compared with protein A apheresis for removal of IgG. The CRP removal was highly specific, as CRP was a predominant protein in the eluted fraction $(5-15 \%$ in the elution fractions vs. $0.17 \%$ in serum; enrichment factor: $29-88$ ). Contaminating proteins were mostly serum albumin and antibodies since we did not perform a baseline wash but a replacement of plasma by physiological sodium chloride solution. Each regeneration circle resulted in a slight plasma loss due to commingling of plasma with saline and the necessity to avoid a volume overload of the animal. The plasma loss will be reduced because the capacity of the adsorber will allow a reduction in regeneration circles in further experiments.

Compared to sham-treated animals, CRP apheresistreated animals had a reduced CRP level persisting also to the day following apheresis. Nonetheless, repeated and controlled apheretic treatment is recommended due to the upregulated CRP synthesis induced by infarction that persists for several days.

For the first time, we were able to show that it is possible to conduct apheresis at the following 2 days after AMI in a porcine infarction model. The infarct resulted in an elevation of CRP, and CRP apheresis temporarily reduced CRP concentration during each apheresis session. This model is suitable to test the importance of different proteins, mediators, etc. during the first days after AMI by specific depletion. The experimental setting will be useful to answer open questions regarding the pathophysiology after AMI, especially with respect to the acute-phase protein CRP and its function as a determinant of infarct size. 


\section{Acknowledgements}

The work was supported by a grant designated to A.S. from the German Federal Ministry of Education and Research in the GOBio program (grant No. 0315095). Adsorber cartridges were kindly provided by Fresenius Medical Care. We gratefully acknowl- edge medicap clinic $\mathrm{GmbH}$ for the copious cooperation, including the generation of a specific software module to run a regenerative single adsorber system on their apheresis control units. We are very thankful to the dialysis nurses from ward 42 of the Charité at CVK, for their assistance with apheresis and to the staff of the animal facility, Charité, for the care for our animals.

\section{References}

1 Casas JP, Shah T, Hingorani AD, Danesh J, Pepys MB: C-reactive protein and coronary heart disease: a critical review. J Intern Med 2008;264:295-314.

2 Nijmeijer R, Lagrand WK, Lubbers YT, Visser CA, Meijer CJ, Niessen HW, Hack CE: Creactive protein activates complement in infarcted human myocardium. Am J Pathol 2003;163:269-275.

3 Pepys MB, Hirschfield GM: C-reactive protein: a critical update. J Clin Invest 2003;111: 1805-1812.

4 Griselli M, Herbert J, Hutchinson WL, Taylor KM, Sohail M, Krausz T, Pepys MB: Creactive protein and complement are important mediators of tissue damage in acute myocardial infarction. J Exp Med 1999;190: 1733-1740.

5 Pepys MB, Hirschfield GM, Tennent GA, Gallimore JR, Kahan MC, Bellotti V, Hawkins PN, Myers RM, Smith MD, Polara A, Cobb AJ, Ley SV, Aquilina JA, Robinson CV, Sharif I, Gray GA, Sabin CA, Jenvey MC, Kolstoe SE, Thompson D, Wood SP: Targeting C-reactive protein for the treatment of cardiovascular disease. Nature 2006;440: 1217-1221.

6 de Beer FC, Baltz ML, Munn EA, Feinstein A, Taylor J, Bruton C, Clamp JR, Pepys MB: Isolation and characterization of C-reactive protein and serum amyloid $\mathrm{p}$ component in the rat. Immunology 1982;45:55-70.
7 Whitehead AS, Zahedi K, Rits M, Mortensen RF, Lelias JM: Mouse C-reactive protein. Generation of cDNA clones, structural anal$y$ sis, and induction of mRNA during inflammation. Biochem J 1990;266:283-290.

8 Duchene DA, Gallagher BL, Ratliff TL, Winfield HN: Systemic and cell-specific immune response to laparoscopic and open nephrectomy in porcine model. J Endourol 2008;22: 113-120.

9 Ho DW, Fan ST, To J, Woo YH, Zhang Z, Lau C, Wong J: Selective plasma filtration for treatment of fulminant hepatic failure induced by $\mathrm{D}$-galactosamine in a pig model. Gut 2002;50:869-876.

10 Sauer M, Altrichter J, Kreutzer HJ, Logters T, Scholz M, Noldge-Schomburg G, Schmidt R, Mitzner SR: Extracorporeal cell therapy with granulocytes in a pig model of Gram-positive sepsis. Crit Care Med 2009;37:606-613.

11 Kim RJ, Judd RM, Chen EL, Fieno DS, Parrish TB, Lima JA: Relationship of elevated $23 \mathrm{Na}$ magnetic resonance image intensity to infarct size after acute reperfused myocardial infarction. Circulation 1999;100:185192.

12 Aschenbach JR, Seidler T, Ahrens F, Schrodl W, Buchholz I, Garz B, Kruger M, Gabel G: Luminal salmonella endotoxin affects epithelial and mast cell function in the proximal colon of pigs. Scand J Gastroenterol 2003;38:719-726.

13 Heegaard PM, Pedersen HG, Jensen AL, Boas U: A robust quantitative solid phase immunoassay for the acute phase protein C-reactive protein (CRP) based on cytidine $5^{\prime}$ diphosphocholine coupled dendrimers. J Immunol Methods 2009;343:112-118.
14 Greenfield JR, Samaras K, Jenkins AB, Kelly PJ, Spector TD, Gallimore JR, Pepys MB, Campbell LV: Obesity is an important determinant of baseline serum C-reactive protein concentration in monozygotic twins, independent of genetic influences. Circulation 2004;109:3022-3028.

15 Orn S, Manhenke C, Ueland T, Damas JK, Mollnes TE, Edvardsen T, Aukrust P, Dickstein $\mathrm{K}$ : C-reactive protein, infarct size, microvascular obstruction, and left-ventricular remodelling following acute myocardial infarction. Eur Heart J 2009;30:1180-1186.

16 Kitsis RN, Jialal I: Limiting myocardial damage during acute myocardial infarction by inhibiting C-reactive protein. $\mathrm{N}$ Engl J Med 2006;355:513-515.

17 Hershcovici T, Schechner V, Orlin J, Harell D, Beigel Y: Effect of different LDL-apheresis methods on parameters involved in atherosclerosis. J Clin Apher 2004;19:90-97.

18 Hovland A, Hardersen R, Sexton J, Mollnes TE, Lappegard KT: Different inflammatory responses induced by three LDL-lowering apheresis columns. J Clin Apher 2009;24: 247-253.

19 Koll RA, Klinkmann J, Richter WO: Rheosorb: A specific adsorber for fibrinogen elimination in clinical situations with impaired rheology. Artif Organs 2002;26:145-151. 